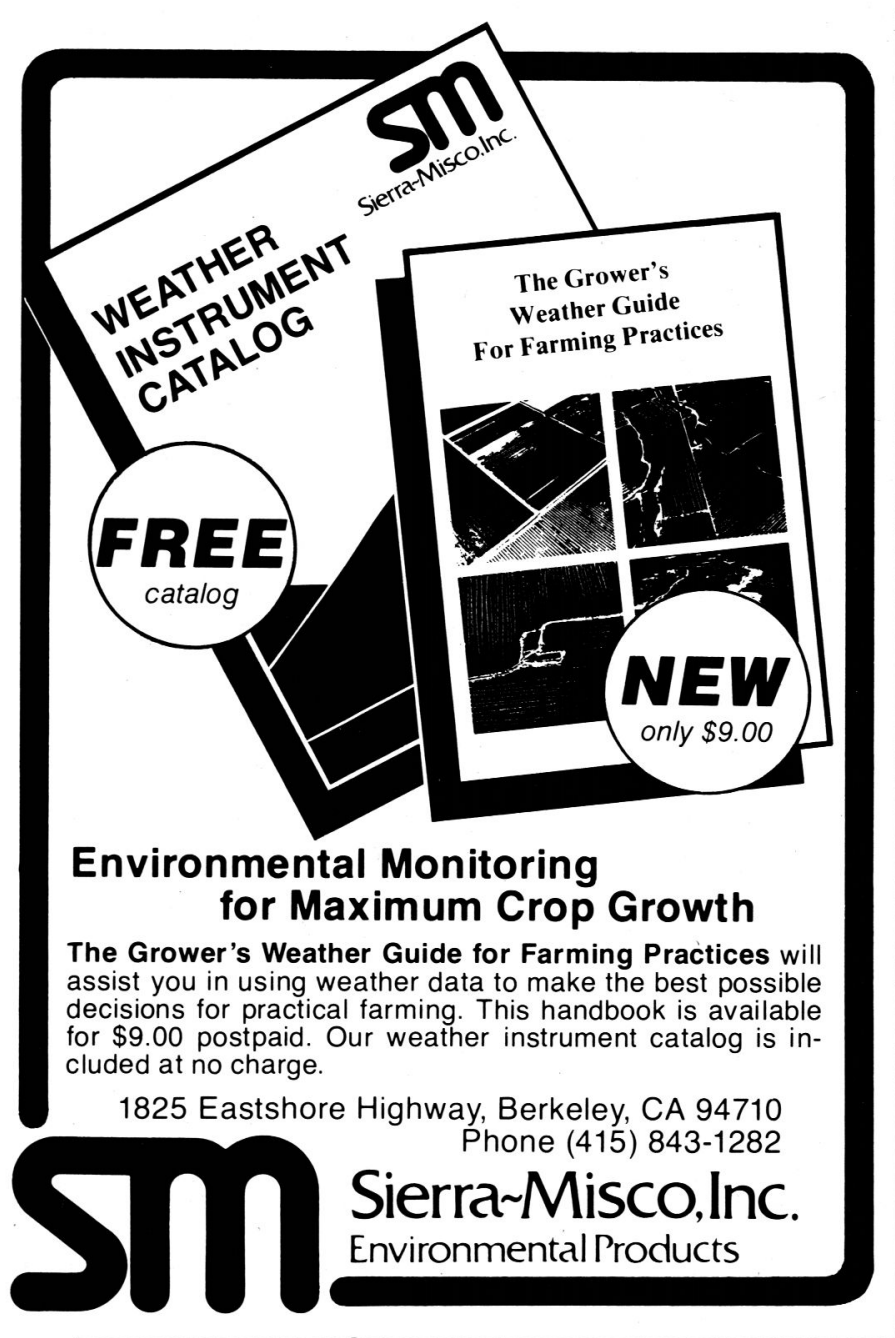

\title{
The Atmospheric Consequences of Nuclear Warfare
}

\author{
A Statement of the Council of the American \\ Meteorological Society as adopted on \\ September 30, 1983
}

Recognizing the inevitable, widespread, devastating consequences of nuclear war by direct explosive effects, and by effects propagated through the atmosphere to the entire globe that could cause the destruction of the biological base that sustains human life, the Council of the American Meteorological Society calls on the nations of the world to take whatever steps are necessary, such as the adoption of appropriate treaties to prevent the use of nuclear weapons and avoid nuclear war.

announcements (continued from page 1272)

"User's Network for Applied Modeling of Air Pollution" (UNAMAP), is a compilation of 31 air quality dispersion models which simulate the distribution and concentration of pollutants in the atmosphere from a wide variety of sources. Based on input of emission and meteorological data, the models mathematically simulate the transport and dispersion of emitted air pollutants. A variety of models are available to be used for different types of sources and pollutant averaging times for both rural and urban situations.

Copies of the UNAMAP systems with associated users manuals are available as PB83-244 368, for $\$ 1115.00$ from NTIS, Computer Products Support Group, Springfield, Va. 22161 (tel.: 703-487-4807).

\section{Minority fellowships}

In addition to its science and engineering research associateships, the National Research Council (NRC) will also be awarding approximately 35 Postdoctoral Fellowships for Minorities in a program designed to provide opportunities for continued education and experience in research to American
Indians, Alaskan Natives (Eskimo or Aleut), Black Americans, Mexican Americans/Chicanos, and Puerto Ricans. Fellowship recipients will be selected from among scientists, engineers, and scholars in the humanities who show greatest promise of future achievement in academic research and scholarship in higher education.

In this national competition, sponsored by the The Ford Foundation, United States citizens who are members of one of the designated minority groups; who are preparing for, or already engaged in college or university teaching; and who hold doctoral degrees may apply for a one year fellowship award.

Awards will be made in the areas of behavioral and social sciences, humanities, EMP fields (engineering sciences, mathematics, physical sciences), life sciences, and for interdisciplinary programs of study. Awards will not be made in professions such as medicine, law, or social work, or in areas such as educational administration, curriculum supervision, personnel, and guidance. Tenure of fellowship provides postdoctoral research experience at an appropriate nonprofit institution of 


\section{A total image processing solution should be affordable, too. The SYSTEM 575 is.}

The $I^{2}$ S System 575 matches a high-performance, general purpose image array processor with a comprehensive library of applications software to form what is truly a "total system." Affordably priced, it's a system delivering solutionsnot new problems - to the entire spectrum of image processing users.

With prices as low as $\$ 75,000$, the System 575 is available as a standalone workstation integrating the powerful Model 75 Image Processor, a DEC PDP-11/23 computer under RSX-11M with disk and magnetic tape, a complete image data base management system and applications software library. Or the System 575 software and Model 75 Processor can be teamed with a variety of minicomputers-including other PDP-11s ${ }^{\star}$, the VAX-11^, HP3000 Series II and III, MC68000 and SEL - at $30 \%$ less cost than any system even coming close in performance. Either way you get instant productivity-affordably!

Unique system "friendliness":

- Simple COMMAND INTER-

PRETER call-up and control of applications software.

- Menu-driven control of 30 interactive applications programs.

- On-line, easily accessed documentation system.

- Automated recall of interactive display functions.

- Instant review of Look-up Table, Function Memory and Register contents.

- Complete "session" history.

- Instant access to Image and

Function Name directories.

- Non-fatal error handling.

\section{Comprehensive software capabilities}

The System's modular software packages are organized into Fundamental Operating Software for the Model 75 Image Processor, a complete Image Data Base Management System with Command Interpreter and the Appli- cations Software Library. General purpose modules include:

- The BASIC MODULE which, combining the Fundamental Operating Software and the Primitives, offers 32 applications programs and the basis for building 60 others.

- The INTERMEDIATE MOD$U L E$, with 37 applications programs extending capabilities to provide general purpose image processing functions.

- The ADVANCED MODULE, with 39 applications programs incorporating complex, compute-intensive image processing techniques.

- The I/O DEVICES MODULE that supports high-resolution, system-oriented image I/O devices.

- Various ADD-ON MODULES for specific applications areas, such as EARTH RESOURCES, are also available.

The Model 75 Processor-fast, powerful, proven

Modular for customizing to stringent user needs, the Model 75 supports a wide range of image

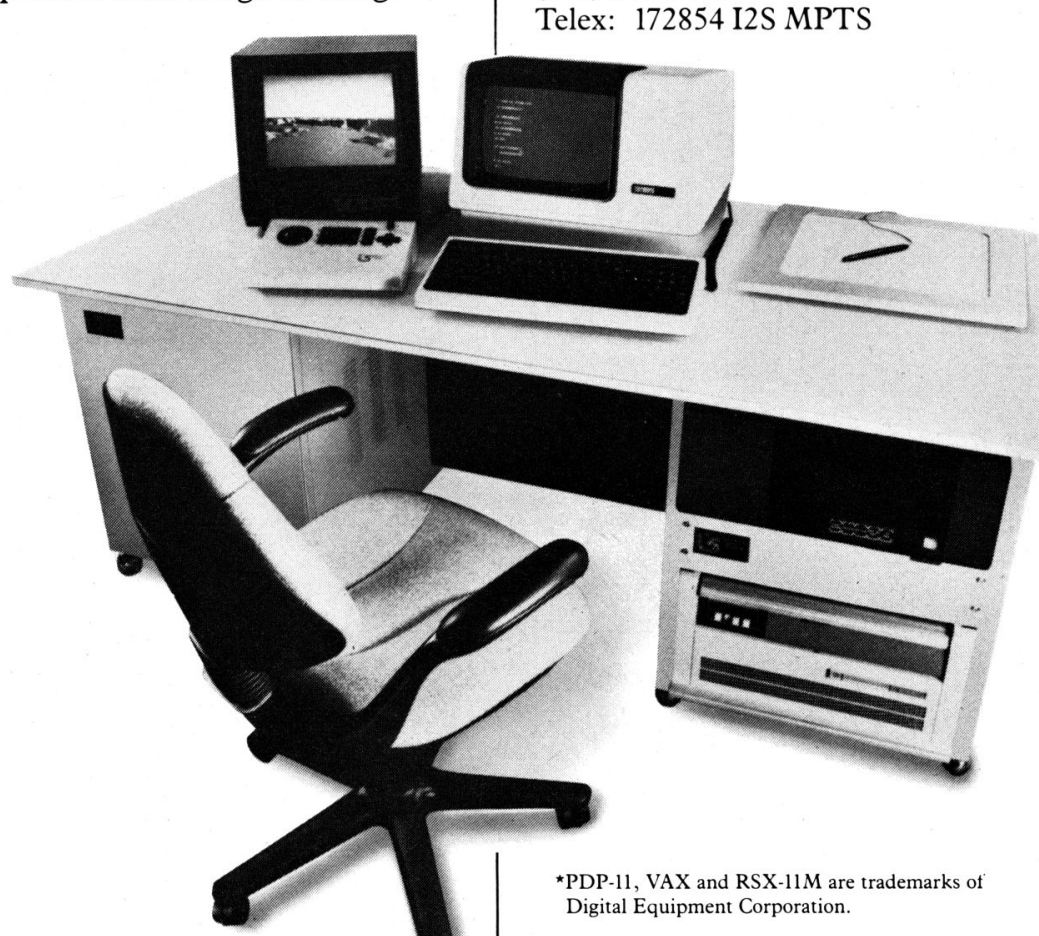

I/O devices and offers features that include:

- Up to 4 MBytes of image refresh memory using $64 \mathrm{~K}$ RAMs.

- Parametric Memory Addressing for defining and controlling refresh memory access.

- 32 Look-up Tables in each pipeline for four levels of "local-area" point processing in each color.

- Independent $\mathrm{X}$ and $\mathrm{Y}$ hardware zoom and scroll for each refresh memory.

- Up to sixteen $512 \times 512 \times 1$-bit planes of graphics overlay memory.

Write or phone today. And learn more about why the "total image processing solution" is also the best performing-and most affordable-system available.

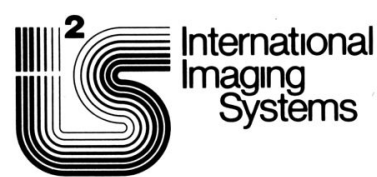

1500 Buckeye Dr., Milpitas, CA 95035 (408) 262-4444 Digital Equipment Corporation. 
